\title{
Experimental and computational studies of jamming
}

\author{
Chaoming Song, Ping Wang, Fabricio Potiguar, Hernán A. \\ Makse $\S$ \\ Levich Institute and Physics Department \\ City College of New York \\ New York, NY 10031, US
}

\begin{abstract}
Jamming is a common feature of out of equilibrium systems showing slow relaxation dynamics. Here we review our efforts in understanding jamming in granular materials using experiments and computer simulations. We first obtain an estimation of an effective temperature for a slowly sheared granular material very close to jamming. The measurement of the effective temperature is realized in the laboratory by slowly shearing a closely-packed ensemble of spherical beads confined by an external pressure in a Couette geometry. All the probe particles, independent of their characteristic features, equilibrate at the same temperature, given by the packing density of the system. This suggests that the effective temperature is a state variable for the nearly jammed system. Then we investigate numerically whether the effective temperature can be obtained from a flat average over the jammed configuration at a given energy in the granular packing, as postulated by the thermodynamic approach to grains.
\end{abstract}

$\S$ To whom correspondence should be addressed (makse@mailaps.org) 


\section{Introduction}

The application of ideas from equilibrium statistical mechanics to nonequilibrium systems has been extensively debated in the literature [1, 2, 3, 4] and has found examples in structural glasses [5], colloidal suspensions [6], spin glasses [7, highly agitated granular matter [8] and a fluidized particle [9].

It has been suggested that, under certain experimental conditions, a jammed granular system could be amenable to a statistical mechanics formulation [10. This requires new statistical concepts specific to this kind of a dissipative athermal system. Provided that all the jammed configurations of the system are equally probable and bear no memory of their creation, we arrive at the ergodic hypothesis, implying that a statistical mechanics approach is justified. This forms the basic tenet of the statistical mechanics formulation for jammed granular matter, which characterizes the packing state by the entropy, $S$, and the compactivity, $X$, defined as $X=\partial V / \partial S$, where $V$ is the system volume [10, 11]. The existence of jammed reversible states in granular matter has been suggested by compaction experiments employing tapping, oscillatory compression or sound propagation as the external perturbation [12, 13, 14, 15, 16.

Recent studies indicate that out-of-equilibrium systems showing slow relaxation dynamics, such as an aging glass or a slowly sheared granular material close to jamming, can be characterized by an "effective temperature" arising from an extension of the fluctuation-dissipation relation to nonequilibrium situations [17. Whereas the theoretical concept of compactivity has been developed for static jammed configurations of grains, the analogy with dynamical measurements of effective temperatures may exist if the motion is sufficiently slow. In fact, the resulting effective temperature is closely related to the compactivity $X$, as obtained by a flat average over the available jammed configurations. Until present, the only evidence for the significance of these ideas in describing granular matter has emerged from theoretical mean-field models [18] and computer simulations of glassy systems and granular matter [19, 20, 21, 22, 23. Macroscopic variables, such as the effective temperature for granular matter, have not been previously measured in the laboratory. This line of research has led to the design of the experiment which we are about to describe.

In this paper we first present experimental evidence [24] for the validity of the effective temperature in describing a slowly sheared granular material very close to jamming. The particle trajectories in the sheared system yield the diffusivity and the mobility from which the temperature is deduced using a fluctuation-dissipation relation. All the particles equilibrate at the same temperature, which is in turn independent of

the slow shear rate, thus suggesting the condition of a state variable for the jammed system. In the second part of this paper we review our efforts [23, 25] to understand the meaning of the effective temperature from a thermodynamic point of view under the framework of the statistical mechanical approach introduced by Edwards [10]. 


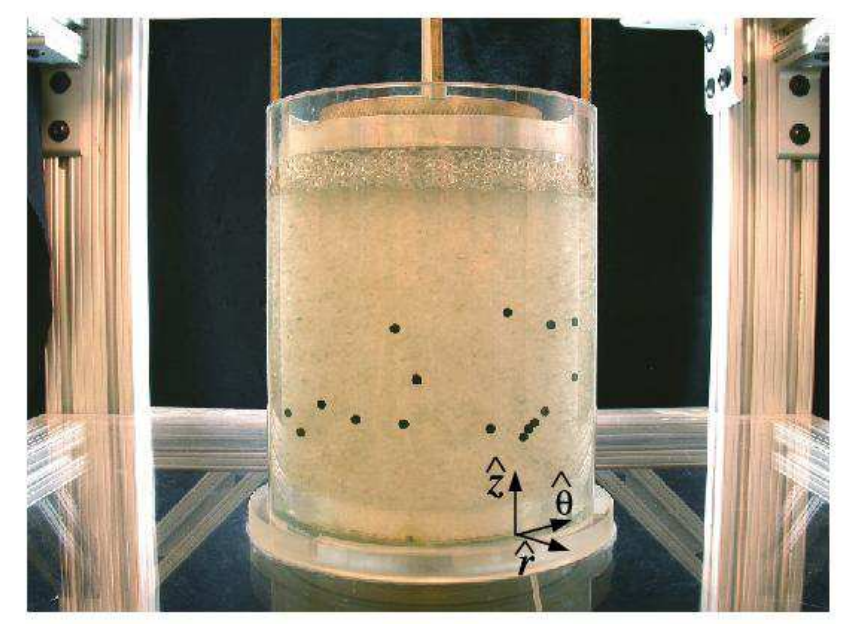

(a)

(b)

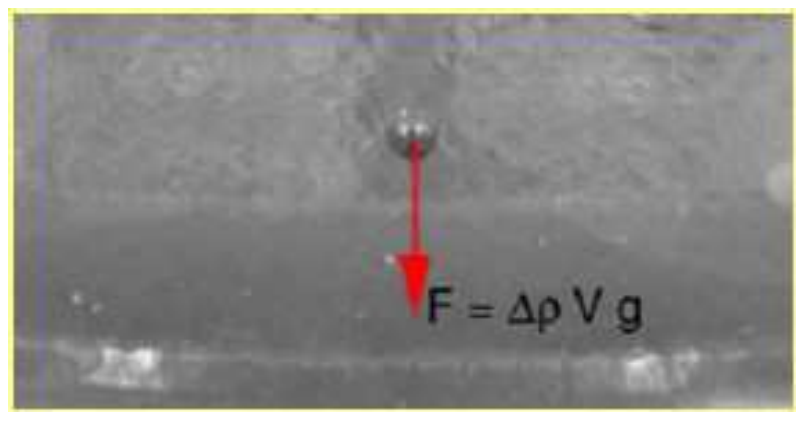

Figure 1. Experimental set-up. (a) Transparent acrylic grains and black tracers in a refractive index and density matched solution are confined between the inner cylinder of radius $50.8 \mathrm{~mm}$ and the outer cylinder of radius $66.7 \mathrm{~mm}$. (b) Detail of a metallic tracer in the background of PMMA particles.

\section{Experiments}

\subsection{Experimental set-up}

The experimental test for the application of statistical mechanics concepts to jammed matter involves using observational techniques to monitor the evolution of the particulate packing as it explores the available configurations. The different packing configurations are investigated using quasi-static shear in a Couette cell geometry, depicted in Fig. 1]

The particulate system is confined between an inner and an outer cylinder. The inner cylinder of the cell is slowly rotated by a motor while the outer cylinder is fixed and transparent for visualization. The walls of both cylinders are roughened by gluing a layer of particles to provide shear motion to the assembly, avoiding wall-slip. The grains 
are compactified by the application of an external pressure of a specific value (typically $386 \mathrm{~Pa}$ ), introduced by a moving piston at the top of the granular matrix. We use a narrow gap Couette cell of the order of 7 particle diameters wide in order to avoid the formation of bulk shear bands [26, 27, 28, 29, 30].

Since a granular assembly is optically impenetrable by its very nature the first task consists in creating a transparent sample. This is achieved by refractive index matching transparent particles with a suitable suspending solution. The presence of the solution reduces friction between the particles, nevertheless the ensemble remains jammed throughout the experiment by the application of the external force via the piston. It is important to note that the liquid only partially fills the cell (see Figure 11). In this way the pressure of the piston is transmitted only to the granular particles and not to the fluid. The key feature of the system is being closely packed, which is hereby satisfied. The random motion of the particles is due to the 'jamming' forces exerted by the enduring contacts of all the neighboring particles which renders the problem nontrivial.

The successful packing consists of a 1:1 mixture of two different sizes of spherical Poly-methyl methacrylate (acrylic) particles, of density $\rho=1.19$ and index of refraction $n=1.49$. We use two different packings, containing either $3.17 \mathrm{~mm}$ and $3.97 \mathrm{~mm}$ diameter particles (Packing 1) or particles of diameter $3.97 \mathrm{~mm}$ and $4.76 \mathrm{~mm}$ (Packing 2 ). The size ratio ensures that crystallization is avoided. The mixture of particles is immersed in a solution of approximately $74 \%$ weight fraction of cyclohexyl bromide and $26 \%$ decalin [31, matching not only the refractive index but more importantly the density of the acrylic particles. The density matching fluid eliminates pressure gradients associated with gravity in the vertical direction. This step avoids problems encountered in previous tests of compactivity [12] and other effects such as convection and size segregation such as the Brazil nut effect inside the cell [32].

We follow the trajectories of tracer particles in the sample bulk to obtain the diffusion of the tracers and the response function (mobility) to an external force within the structure. These measurements lead to the compactivity or effective temperature via a fluctuation-dissipation relation generalized to granular media. If the resulting effective temperature is a physical variable of the system, it will be independent of the properties of the tracer particles- a necessary but not sufficient condition. It is to this end that we contribute experimental results. We note that to evaluate the broader thermodynamic meaning of the temperature it would be needed to examine whether different measures of temperature agree as well. Such tests could be performed, for instance, by measuring the temperature for different observables in the system.

The tracer particles must experience a constant force, in response to which the mobility can be measured. Tracers of a different density to the acrylic particles are then added to the packing, as shown in Fig. 1. Two types of tracers, made of nylon $(\rho=1.12)$ and delrin $(\rho=1.36)$, are employed. The role of the tracer in the system is to explore the different packing configurations, and the size of the tracers is chosen, accordingly, of similar size as the background particles. If the tracers are much larger or smaller than 


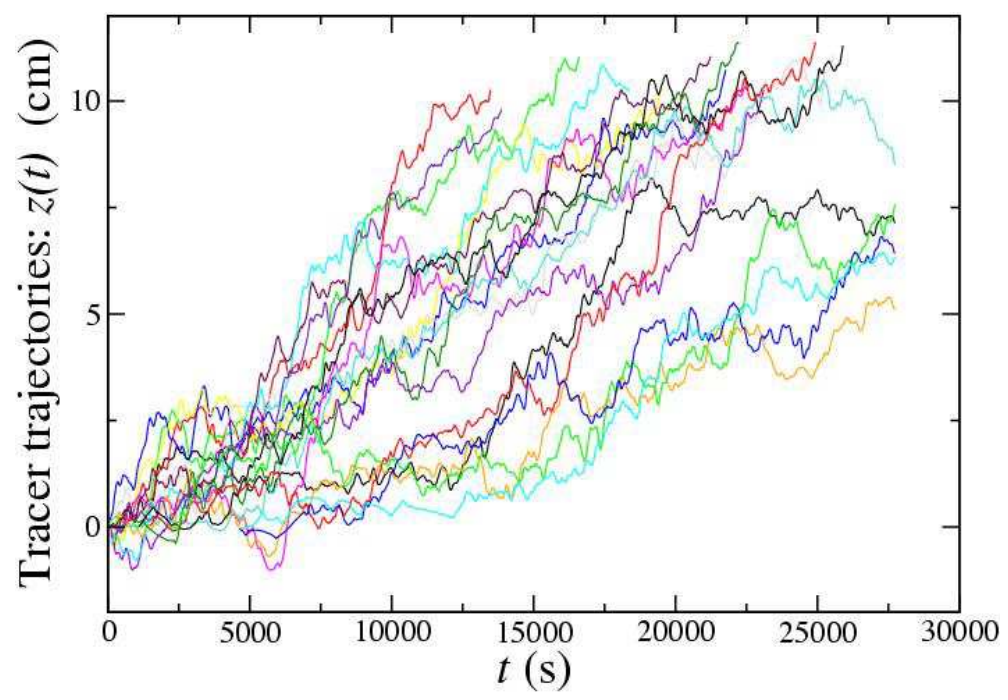

Figure 2. Trajectories of the $3.97 \mathrm{~mm}$ nylon tracers in Packing 1 showing the diffusion and response to the gravitational force when sheared in the Couette cell. Note that the tracers diffuse distances larger than the particle diameter indicating that we are probing fluctuations outside the "cages" formed by the surrounding particles.

the background particles new physics would be brought into the problem. For instance if a tracer is small enough to fall into the voids of the other particles then "percolation effects" 33] would prevail and the displacements would be larger than those predicted by the effective temperature. Such a tracer would no longer prove the background particle network, but would instead have different dynamics and test other interesting effects which cannot be captured by the present formalism.

\subsection{Experimental Results}

The first experiment uses Packing 1 with 20 tracers of $3.97 \mathrm{~mm}$ nylon beads. The Couette cell is sheared at very slow frequencies $f=2.4 \mathrm{mHz}$ defining the external shear-rate $\dot{\gamma}_{e}=2 \pi f r_{1} /\left(r_{2}-r_{1}\right)=0.0481 / \mathrm{s}$, where $r_{1}=50.8 \mathrm{~mm}$ and $r_{2}=66.7 \mathrm{~mm}$ are the radii of the inner and outer cylinders, respectively. We require a very slow shear rate so that the system is close-packed at all times. The $(r(t), \theta(t), z(t))$ coordinates of the tracers are obtained by analyzing the images acquired by four digital cameras surrounding the shear cell $[(r, \theta)$ are obtained only at the overlaps of the cameras]. In the following we first present results for the $z$ direction since this is the only direction where the temperature can be calculated with the present set-up (the external force acts only vertically).

The resulting vertical trajectories of the tracers $z(t)$ are depicted in Fig. 2] showing that the nylon tracers not only diffuse, but also move with a constant average velocity to the top of the cell. We confine the measurements of the tracer fluctuations away from the inner rotating cylinder to avoid boundary effects and where the average tangential velocity of the tracers, $v_{\theta}(r)$, can be approximated linearly as $v_{\theta}(r) \approx-\dot{\gamma}_{l} r$ with a 


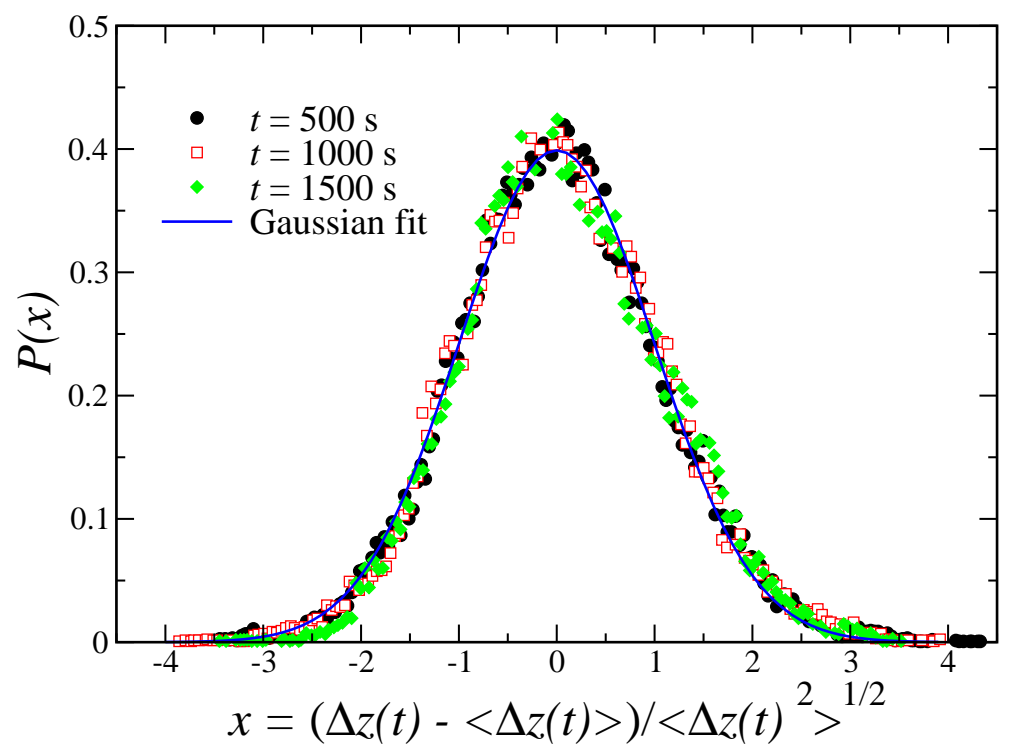

Figure 3. Probability distribution of the displacements of the $3.97 \mathrm{~mm}$ nylon tracers in Packing 1. The different distributions at different times are rescaled according to a Gaussian distribution.

constant local shear rate $\dot{\gamma}_{l}=0.0211 / \mathrm{s}$. This ensures that the diffusivity (which depends on the local shear rate) remains approximately constant in the radial direction.

The statistical analysis of the particle displacements $\Delta z(t)=z\left(t+t_{0}\right)-z\left(t_{0}\right)$ reveals a Gaussian distribution which broadens with time, as seen in Fig. 3. The rms fluctuations grow linearly for sufficiently long times (see Fig. 4a):

$$
\left\langle\left[z\left(t+t_{0}\right)-z\left(t_{0}\right)\right]^{2}\right\rangle \sim 2 D t,
$$

where $D$ is the self-diffusion constant and $\langle\cdots\rangle$ denotes ensemble average over the tracers and over the initial time $t_{0}$. For the $3.97 \mathrm{~mm}$ tracer we obtain $D_{3.97 \mathrm{~mm}}=(1.1 \pm 0.1) \times 10^{-8}$ $\mathrm{m}^{2} / \mathrm{s}$.

Figure $4 \mathrm{~b}$ shows the mean value of the position of the tracers extracted from the peak of the Gaussian distribution as a function of time, thus yielding the mobility $\chi$ as

$$
\left\langle z\left(t+t_{0}\right)-z\left(t_{0}\right)\right\rangle \sim \chi F t .
$$

Here $F=\left(\rho_{a}-\rho_{t}\right) V g$ is the gravitational force applied to the tracers due to their density mismatch while $\rho_{a}$ and $\rho_{t}$ are the densities of the acrylic particles and the tracers respectively, $V$ is the volume of the tracer particle and $g$ the acceleration of gravity. The value of the mobility for the $3.97 \mathrm{~mm}$ tracer is $\chi_{3.97 \mathrm{~mm}}=(9.7 \pm 0.9) \times 10^{-2} \mathrm{~s} / \mathrm{kg}$. We check that the mobility of the tracers is constant in the region of measurements.

An important task is to determine whether there exists a linear response regime in the system, which would imply that the mobility is independent of the external gravitational force as $F \rightarrow 0$. The external force is varied by changing the density of the tracers of the same size, under the assumption that the surface properties remain 


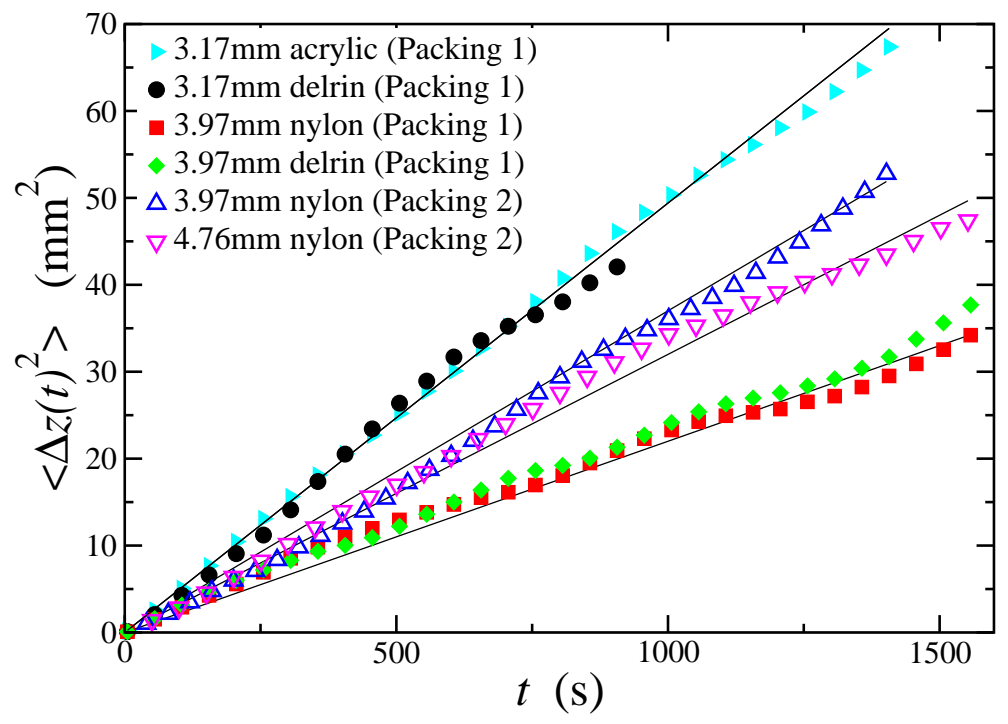

a

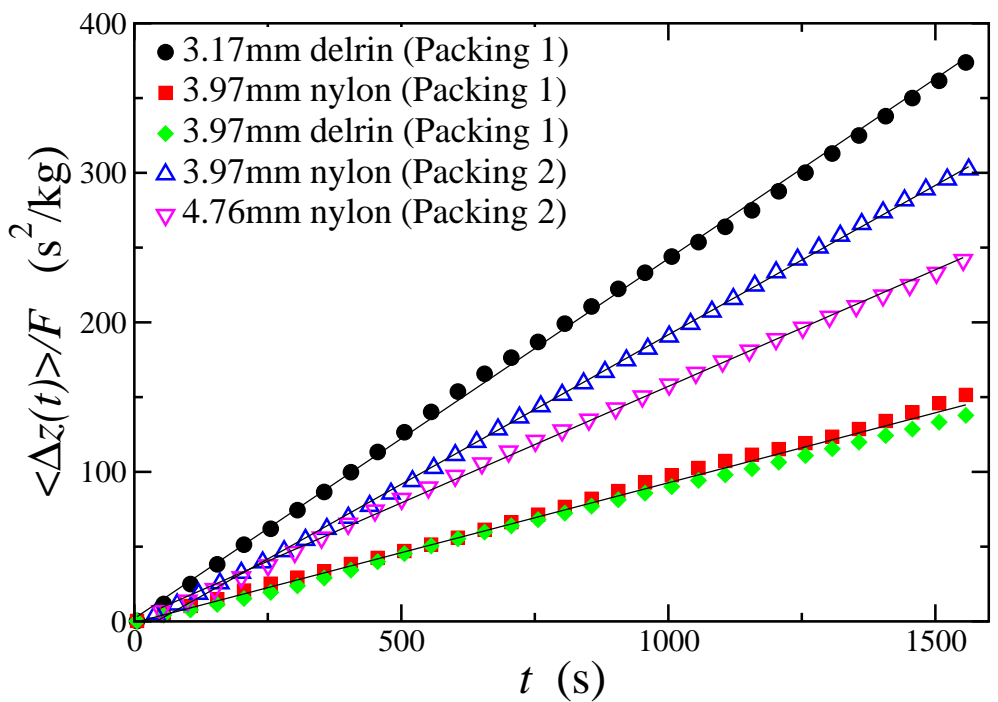

Figure 4. (a) Diffusion and (b) mobility of tracers. We use Packing 1 and Packing 2 of acrylic particles and tracers of different sizes and densities. Packing 2 is run at $\dot{\gamma}_{e}=0.0241 / \mathrm{s}$. For both packings, $D$ and $\chi$ are inversely related to the tracer sizes.

unchanged. This is realized experimentally in Packing 1 by the introduction of delrin tracers of $3.97 \mathrm{~mm}$ diameter, the density of which is higher than that of nylon. The analysis of the trajectories reveals that the mobility is the same for both tracers so that it is independent of the external force, as shown in Fig. 4 $\mathrm{b}$. The use of tracers even heavier than delrin reveals the appearance of nonlinear effects in the mobility. Moreover we show that the diffusion constants of both types of tracers are approximately the same (see Fig. 4a) confirming that the external force on the tracers does not affect the diffusion constant for the small forces used in this study. 


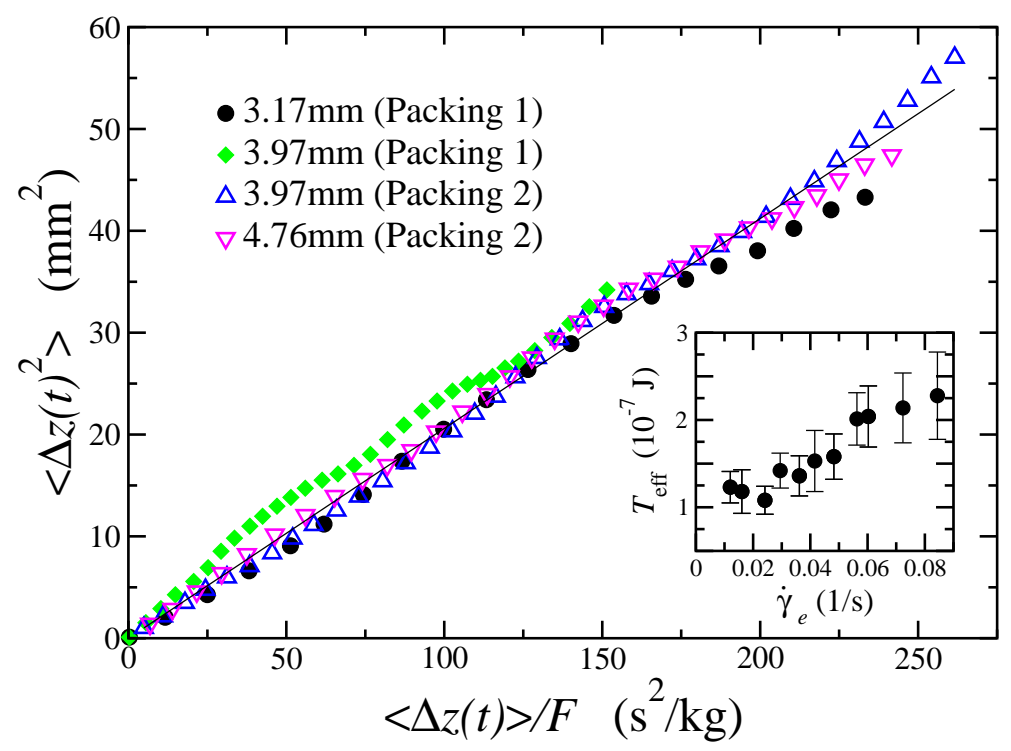

Figure 5. Effective temperatures for various tracers and different packings as obtained from a parametric plot of their diffusion versus mobility, as explained in the text. The slopes of the curves for different tracers consistently yield the same average value of $T_{\text {eff }}=(1.1 \pm 0.1) \times 10^{-7} \mathrm{~J}$ as given by Eq. (3). The inset shows the dependence of $T_{\text {eff }}$ on the shear rate $\dot{\gamma}_{e}$ for the $4.76 \mathrm{~mm}$ nylon tracers in Packing 2. We find that $D \sim \dot{\gamma}_{e}$ and $\chi \sim \dot{\gamma}_{e}$, while $T_{\text {eff }}=D / \chi$ is shear-rate independent for sufficiently small $\dot{\gamma}_{e}$.

\subsection{Effective temperature}

According to a Fluctuation-Dissipation relation, it is the diffusivity and the mobility of the particles which enable the calculation of an effective temperature, $T_{\text {eff }}$, via an Einstein relation for sheared granular matter:

$$
\left\langle\left[z\left(t+t_{0}\right)-z\left(t_{0}\right)\right]^{2}\right\rangle=2 T_{\mathrm{eff}} \frac{\left\langle z\left(t+t_{0}\right)-z\left(t_{0}\right)\right\rangle}{F} .
$$

A parametric plot, with $t$ as a parameter, of the fluctuations and responses is produced to yield the linear relationship shown in Fig. 5, the gradient of which gives $2 T_{\text {eff. }}$ We obtain for the $3.97 \mathrm{~mm}$ tracer $T_{\text {eff }}=(1.1 \pm 0.1) \times 10^{-7} \mathrm{~J}$. This value is set by a typical energy scale in the system [34, for instance $\left(\rho_{a}-\rho_{t}\right) g d$, which is the gravitational potential energy to move a tracer particle a distance of the particle diameter $d$. The corresponding temperature which would arise from the conversion of this energy into a temperature via the Boltzmann constant, $k_{B}$, is $T_{\text {eff }}=2.7 \times 10^{13} k_{B} T$ at room temperature. This large value is expected 34] (and agrees with computer simulation estimates [23]) since granular matter is an athermal system. We notice that previous results [23] suggest that Eq. (3) is equivalent to the average over jammed configurations, then Eq. (3) can be used to obtain an estimate of the compactivity of the packing [10] as $X=T_{\text {eff }}$. 
An important evidence in examining the thermodynamic meaning of the effective temperature can be obtained from a test of the zeroth law. In other words, changing the tracer size should give rise to a different diffusion and mobility but they should nevertheless lead to the measurement of the same effective temperature, if the system is at "equilibrium". We next introduce tracers of $3.17 \mathrm{~mm}$ diameter in Packing 1 and repeat the above calculations. We find that the $3.17 \mathrm{~mm}$ tracers produce a significantly different diffusion and mobility than their $3.97 \mathrm{~mm}$ counterparts as shown in Fig. 4 $\left(D_{3.17 \mathrm{~mm}}=(2.5 \pm 0.3) \times 10^{-8} \mathrm{~m}^{2} / \mathrm{s}\right.$ and $\left.\chi_{3.17 \mathrm{~mm}}=(2.4 \pm 0.3) \times 10^{-1} \mathrm{~s} / \mathrm{kg}\right)$. In all cases $D$ and $\chi$ increase with decreasing size of the tracers. However, the parametric plot of diffusivity versus mobility demonstrates that their effective temperature are the same as seen in Fig. [5] with an average value over all tracers of $T_{\text {eff }}=(1.1 \pm 0.1) \times 10^{-7} \mathrm{~J}$.

We further check that the diffusion is not affected by the external force by calculating the diffusivity of the nontracers particles by dying acrylic tracers and analyzing their trajectories. As shown in Fig. 4a the diffusion of the acrylic tracers of size $3.17 \mathrm{~mm}$ (for which no external force is applied) is the same as the diffusion of the delrin tracers of the same size (for which the gravitational force is applied).

Next we perform another consistent measurement arising from a repeat of the experiment for a different packing of spherical particle (Packing 2). The use of larger particles of approximately the same size ratio as in Packing 1 still leads to the same volume fraction of particles. Since $T_{\text {eff }}$ is a measure of how dense the particulate packing is (i.e. a large $T_{\text {eff }}$ implies a loose configuration, e.g. random loose packing, while a reduced $T_{\text {eff }}$ implies a more compact structure, e.g. random close packing), it holds to reason that it should be the same for both of the packings under investigation. Indeed, despite the change in their respective diffusivities and mobilities as shown in Fig. 4, the two packings measure the same effective temperature shown in Fig. 5.

An important assumption in this study is that of the system being continuously jammed despite the presence of rearrangements under shear. For this reason, we show in the inset of Fig. [5 that the effective temperature is approximately independent of the shear rate, as long as the particulate motion is slow enough such that enduring contacts prevail. We find that $D \sim \dot{\gamma}_{e}$ and $\chi \sim \dot{\gamma}_{e}$, while $T_{\text {eff }}=D / \chi$ remains approximately constant for sufficiently small $\dot{\gamma}_{e}$. It is within this quasi-static range where the effective temperature could be identified with the temperature of the jammed states. This quasistatic regime has been obtained in previous computer simulations [21, and it is shown in the numerical section of this paper. The regime corresponds to the regime where the stress in the system becomes shear-rate independent [35].

Given that we are dealing with an athermal system in which the notion of 'bath' temperature plays no role, but the thermodynamic concept of temperature still holds, perhaps further explanation is required in terms of the actual role of $T_{\text {eff }}$ in describing granular systems. The length scale on which the particles diffuse over the long time scale of the experiment is of the order of several particle diameters (see Fig. 2] and 4a) implying that the exploration of the available jammed configurations takes place by rearrangements of the particles outside their "cages". The trajectory of the slow moving 
system can be mapped onto the successive jammed states that the system explores. This paints a configurational landscape of jamming, analogous to that observed in the inherent structures formalism of glassy materials [36, 3]. Thus, we identify $T_{\text {eff }}$ as the variable governing this exploration of the different jammed configurations. In contrast to the measurement of the temperature of the slow modes, we also measure the temperature of the fast modes as given by the instantaneous rms fluctuations of the velocity of the particles inside each cage. This kinetic granular temperature is smaller than $T_{\text {eff }}$ and differs for each tracer indicating that it is not governed by the same statistics. Similar results have been obtained in experiments of vibrated granular gases [37.

\subsection{Outlook}

In the next section we present our computational efforts to understand jamming. We will treat the question whether the present definition of effective temperature can be related to the thermodynamic formalism of grains. The main assumption of this statistical formulation is that the different jammed configurations are taken to have the same statistical weight [10]. Thus, observables can be obtained as "flat" averages

of the jammed configurations [18, 20, 22, 23. The validity of this assumption has been extensively debated in the literature (see for instance [3]). Some simulations and analytical work suggest that the effective temperature obtained by applying the extension of FDT to out-of-equilibrium systems is indeed analogous to performing a flat average over the configurational space. Numerically it has been suggested that the effective temperature can be identified with the compactivity introduced in [10], arising from the entropy of the packing [18, 20, 23. In the next section we present our studies showing that under some conditions the effective temperature can be related to a temperature obtained from entropic considerations using a flat average over the jammed configurations. We review the results published in [23, 25].

\section{Numerical studies}

This section describes the potential for using computer simulations in testing the thermodynamic foundations raised in the previous sections. Rather than employing rough rigid grains for which most of the theoretical concepts have so far been devised, computer simulations are obliged to introduce some deformability into the constituent particles to facilitate the measurement of the particle interactions with respect to their positions. As a result, the entropic considerations which have been explained only in terms of the volume in the original formulation of Edwards [10] for the case of rigid grains will now be generalised to situations in which there is a finite energy of deformation in the system [1].

The entropy of the jammed packing can be redefined as a function of both energy and volume,

$$
S(E, V)=\lambda \ln \Sigma_{\text {jammed }}(E, V),
$$


where $\Sigma_{\text {jammed }}(E, V)$ measures the number of microstates for a given volume $V$ and energy $E$. The introduction of energy into the system implies a corresponding compactivity,

$$
X_{E}^{-1}=\frac{\partial S}{\partial E}
$$

where the subscript $E$ denotes that the compactivity is the Lagrange multiplier controlling the energy of the jammed configuration, not the volume. Notice that $X_{E}$ differs from the temperature of an equilibrium system $T=\partial E / \partial S$ because the energy in Eq. (15) is the energy of the jammed configurations and not the thermal equilibrium energy. The radical step is the assumption of equally probable microstates which leads to an analogous thermodynamic entropy associated with this statistical quantity.

In the next sections we present results for the effective temperature, defined through the fluctuation-dissipation theorem, of granular material obtained from extensive computer simulations of a sheared granular packing of compressible spheres. We show that this parameter is independent of the shear rate in a defined interval for high volume fractions, and does not depend on the particular species of particle where it is measured. We also compare these measurements with the granular temperature and show that, indeed, the last one is not the thermodynamical parameter for the jammed states. We then show that the temperature can be obtained as a flat average over the jammed configurations. This approach was validated numerically in 23 , who showed that the temperature obtained by performing a flat average over the jammed configuration is the same, within error bars, as the effective temperature obtained dynamically from a fluctuation-dissipation relation in a gently sheared, bi-disperse assembly of granular balls.

\subsection{The system}

We used Molecular Dynamics [38] to model our system. This system is composed of a bi-disperse assembly of 1000 spheres, half large, half small, with radii ratio of $R_{S} / R_{L} \sim 0.818$, which are initially randomly generated in a periodic cubic cell of size $L$. The spheres interact via normal and tangential forces. To describe these interactions we consider two spheres in contact following the contact force model of Hertz-Mindlin 39. The normal force is given by the Hertz model such that the force is proportional to the deformation of the spheres to the $3 / 2$. The tangential force between two spheres in contact is given by Mindlin no-slip solution for the contact. This force is proportional to the magnitude of the normal force and depends on the loading path of contact. During the contact, the tangential force between two grains can increase until it reaches the Coulomb threshold of static friction, $F_{t}=\mu F_{n}$ in which case the grains slide. Gravity is absent in all our simulations. We can also study packings in which the particles interact only by normal forces, and this case will be referred to as the no-friction, or frictionless, case. We notice that the frictionless case would model a system of compressed emulsions 11. 
a) Simulations

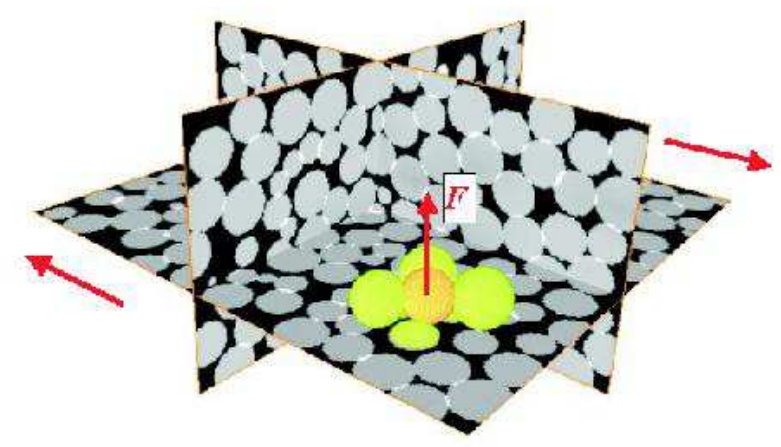

Figure 6. Detail of the simulations of grains of 100 microns interacting via HertzMindlin contact forces. A slow shear flow, indicated by the arrows, is applied to the jammed system. We follow the tracer particle trajectories to obtain the diffusivity. An external force $F$ is then applied to the tracers in response to which we measure the particle mobility. These dynamical measurements yield an effective temperature obtained from an Einstein relation.

Since our goal is to study dense systems, we should compress the initial packing in order to attain a higher volume fraction. It is known [39, 40] that there is a jamming transition at a critical volume fraction $\phi_{c}$ of randon close packing (RCP) above which a disordered solid state persists indefinitely. This metastable state is characterized by a non-vanishing internal pressure and coordination number. Therefore, we choose three initial equilibration pressure values, $P=1,10,100 \mathrm{MPa}$, that are high enough to generate a disordered jammed state. The corresponding initial volume fractions of these states are $\phi=0.6428,0.6623,0.7251$, above the RCP volume fraction. We also consider systems below the jamming transition in the "fluid-like" state for volume fractions $\phi=0.6318,0.6104,0.5999<\phi_{c}$.

We consider a system of large and small spherical grains in a periodic cell. The simulations involve the application of a gentle shear on the particles in the $x-y$ plane, at constant volume (see Fig. [6), with $x$ the direction of the flow and $y$ the velocity gradient. We follow the trajectory of the particles in the $z$-vorticity direction. We used a modified version of the usual Lees-Edwards boundary conditions which imposes a linear velocity profile in the shear plane. Normal periodic boundary conditions are enforced in the $z$-direction. We focus our study on the region of slow shear rates, where the system is always close to jamming. We avoid shear bands by imposing a linear velocity profile and avoid segregation which may occur at much longer time scales than those employed in our computations. 


\subsection{Diffusion, mobility and effective temperature}

We calculate the effective temperature, $T_{\text {eff }}$, of the granular system by measuring it through the same FDT as in the experiments, via diffusion and mobility.

The calculation of the diffusion coefficient involves the square displacement averaged over all particles and over time. All quantities which depend on the Cartesian coordinates will be evaluated in the direction perpendicular to the shear plane, in our case $z$-direction, in order to avoid complications with shear-induced displacements. The mobility of a particle, $\chi$, is the linear response function associated with the motion of this particle under an applied external force. To calculate $\chi$, a small constant force must be applied to the particle in order to induce a displacement in $z$-direction. The effective temperature is defined from the Einstein relation between $D$ and $\chi$ as in Eq. (3).

We plot in Fig. $7 T_{\text {eff }}$ as a function of the shear rate $\dot{\gamma}$ for different pressures and volume fractions. We can see that at higher volume fractions, in no-friction and friction cases, this effective temperature is approximately independent on the shear rate. This is an important result for it shows that this is indeed an intrinsic property of the jammed system, since it does not depend on the driving strength. The jammed state for $\phi>\phi_{c}$ can be characterized by the independence of the effective temperature on the shear rate since this behaviour is not observed in the other cases studied.

Next we perform a similar test of the zeroth-law as done experimentally. We test this by repeating the mobility and diffusion calculations with the small particles for two system of frictionless grains at $1 \mathrm{MPa}$ and $10 \mathrm{MPa}$. Figure 8 shows the plot of diffusion versus mobility for the large and small frictionless particles at 1 and $10 \mathrm{MPa}$, sheared at $\dot{\gamma}=5 \times 10^{-6}$. The agreement is clearly excellent. This further suggests that $T_{\text {eff }}$ is a good thermodynamic variable for the system.

Finally, we compare the effective temperature with the kinetic granular temperature as done in the experiments. As in thermal systems, we can define a parameter called kinetic granular temperature, $T_{k}$, which is the average of the kinetic energy. This is an estimate of the temperature of the fast modes of the system (since a granular system is athermal by definition) and, in general, should not be equal to the effective temperature.

Our numerical results confirm that $T_{\text {eff }}$ is always larger than the granular temperature, $T_{k}$. We also obtain that the behavior of $T_{\text {eff }}$ with volume fraction is different from the behaviour of $T_{k}$ in $\phi$. The last one decreases with increasing $\phi$ while the former increases. This implies that the effective temperature is the temperature of the slow modes, while the granular temperature is the temperature of the fast modes of our system, and the last one is not the temperature for the jammed state.

The next crucial test for this assumption is to show that the effective temperature obtained dynamically can also be obtained via a flat average over the jammed configurations. Such a test has been performed in [23], where it was indeed shown that $T_{\text {eff }}$ is very close to the compactivity of the packing $X_{E}$. This result will be shown explicitly in the next section. We conclude that the jammed configurations explored during shear are sampled in an equiprobable way as required by the ergodic principle. 

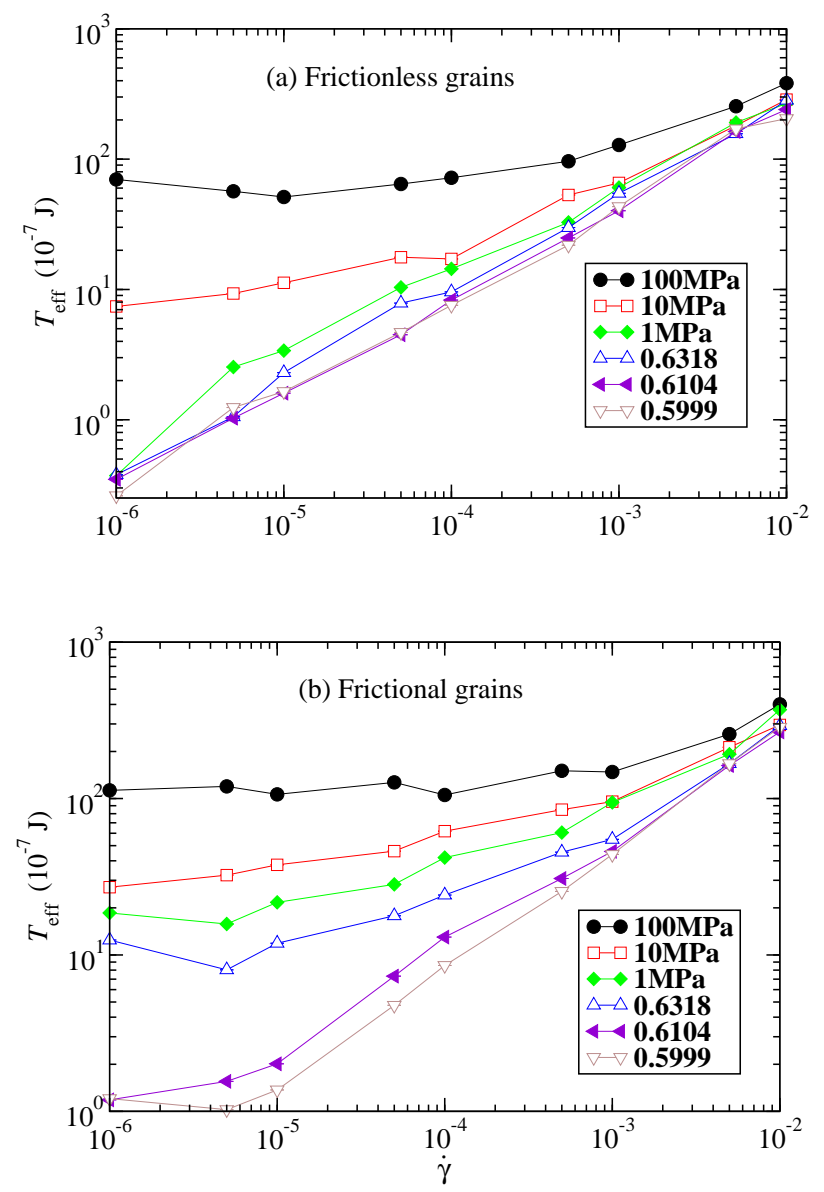

Figure 7. Effective temperature versus shear rate for (a) frictionless particles and for (b) frictional particles. We consider systems above the jamming transition for pressures $p=1 \mathrm{MPa}, 10 \mathrm{MPa}$, and $100 \mathrm{MPa}$, and below the jamming transition for volume fractions $\phi=0.6318,0.6104$, and 0.5999 . For the frictionless case, the independence of $T_{\text {eff }}$ with the shear rate is only observed in the 10 and $100 \mathrm{MPa}$ cases. The other states seem to be unstable under shear. For frictional particles, the effective temperature is approximately independent with the shear rate even in the $\phi=0.6318$ case. This is evidence that the jammed state could be attained by a granular system without any initial compression.

Moreover the dynamical measurement of compactivity renders the thermodynamic approach amenable to experimental investigations as done in the first part of this review.

In the next section we show the relation between the effective temperature of the packing obtained dynamically and the compactivity calculated employing a configurational average.

\subsection{Exploring the jammed configurations via a flat average. Test of ergodicity: $T_{\text {eff }}=X_{E}$}

The systems under investigation have exponentially large (in the number of particles) number of stable states jammed at zero bath temperature. In the previous section we explored such an energy landscape via slow shear. Next, we develop an independent 


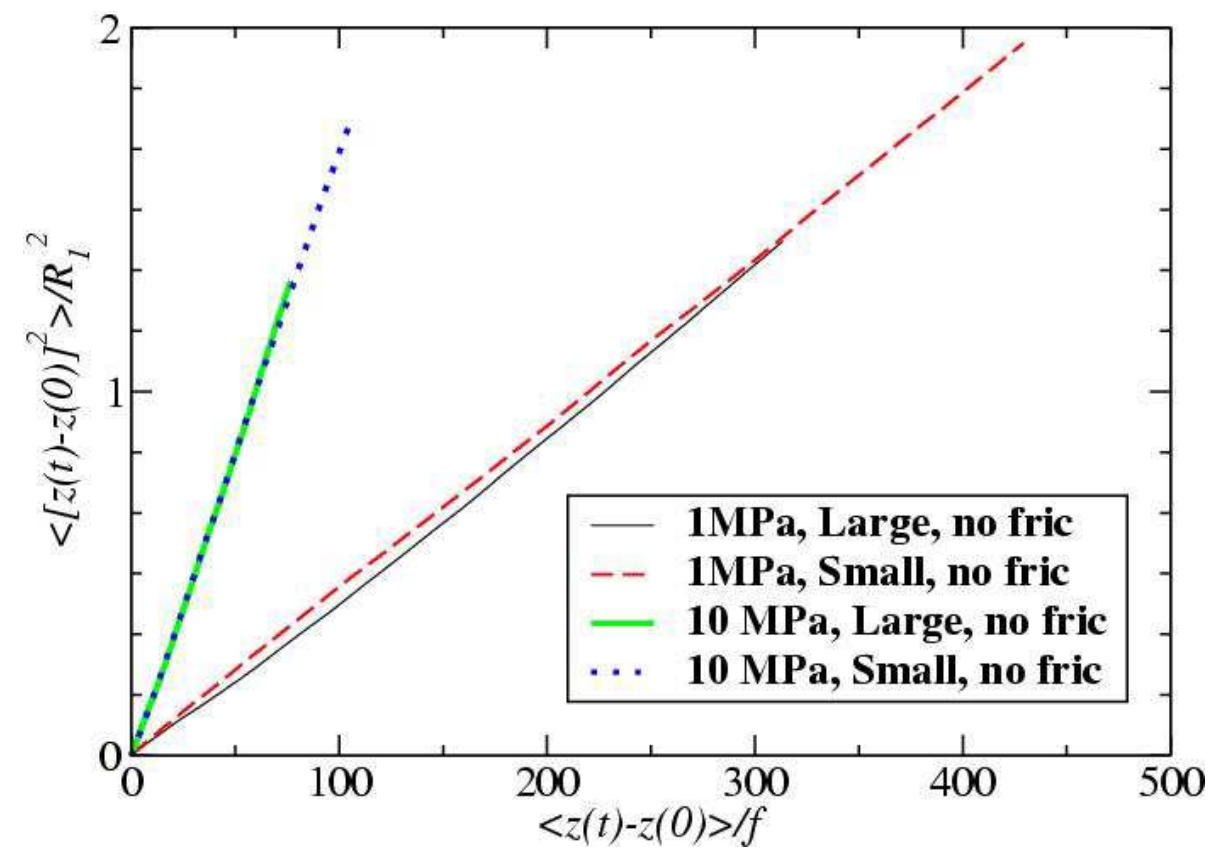

Figure 8. Parametric plot of diffusion coefficient versus mobility. The equality of the effective temperatures for both species of particles is evident in this plot. This gives another evidence that $T_{\text {eff }}$ is an intrinsic property of the system. The legend displays the equilibrated pressure, the size of the particles and if they have friction or not. The curves for the $10 \mathrm{MPa}$ case overlap almost perfectly.

method to study the configurational space. It allows us to investigate the statistical properties of the jammed states available at a given energy and volume. In turn we investigate whether it is possible to relate the dynamical temperature obtained above via a diffusion-mobility protocol to the configurational compactivity based on a flat average over jammed states.

In order to calculate $X_{\mathrm{E}}$ and compare with the obtained $T_{\text {eff }}$ we need to sample the jammed configurations at a given energy and volume in a equiprobable way. In order to do this we sample the jammed configurations with the following probability distribution:

$$
P_{\nu} \sim \exp \left[-E^{\nu} / T^{*}-E_{\text {jammed }}^{\nu} / T_{\text {aux }}\right]
$$

We consider only frictionless grains for this calculation, so the deformation energy $E$ corresponds to the Hertzian energy of deformation of the grains. The extra term added in Eq. (6) allows us to perform the flat sampling of the jammed states. The jammed energy is such that it vanishes at the jammed configurations:

$$
E_{\text {jammed }} \propto \sum_{a}\left|\vec{F}_{a}\right|^{2}
$$

where $\vec{F}_{a}$ is the total force exerted on particle $a$ by its neighbours.

We introduce two "bath" temperatures which will allow us to explore the configuration space and calculate the entropy of the packing assuming a flat average over the jammed configurations. We perform equilibrium MD simulations with two auxiliary "bath" temperatures $\left(T^{*}, T_{\text {aux }}\right)$, corresponding to the partition function (6). 


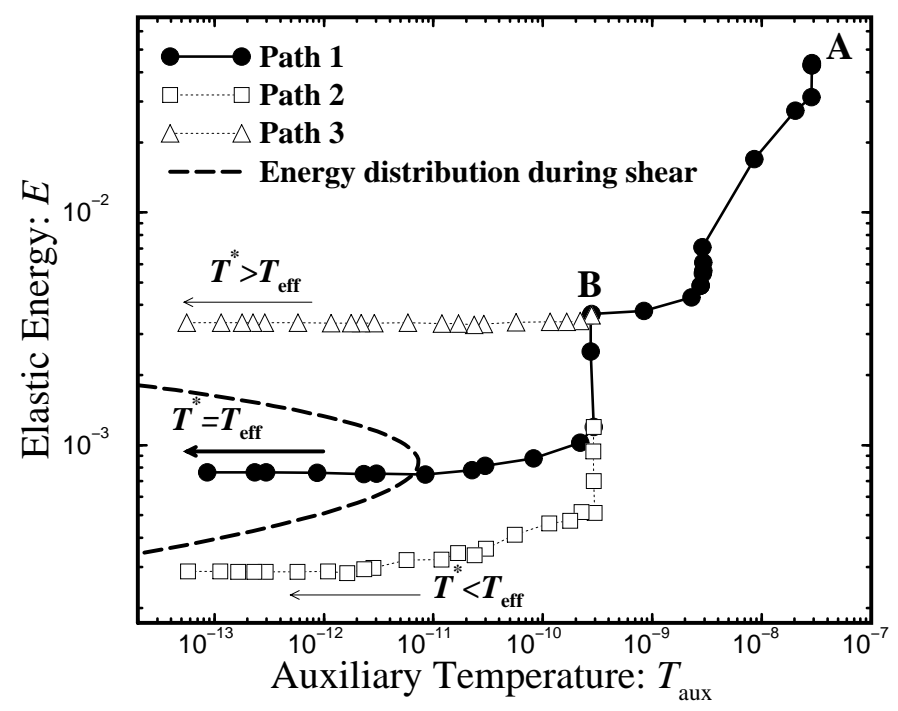

Figure 9. Annealing procedure to calculate $X_{E}$ at different elastic compressional energies. For this calculation we use a system of 200 frictionless particles. The small system size is needed due to the intensive calculations required by the annealing procedure. We plot the elastic energy vs $T_{\text {aux }}$ during annealing together with the distribution of elastic energies obtained during shear (dashed curve, mean value $\left.\langle E\rangle=8.4 \times 10^{-4}\right)$. We equilibrate the system for $40 \times 10^{6}$ iterations at A: $\left(T^{*}=\right.$ $\left.3.4 \times 10^{-2}, T_{\text {aux }}=3 \times 10^{-8}\right)$. We then anneal slowly both temperatures until B: $\left(T^{*}=3.4 \times 10^{-4}, T_{\text {aux }}=3 \times 10^{-10}\right)$, where we split the trajectory in three paths in the $\left(T^{*}, T_{\text {aux }}\right)$ plane. Path 1: we anneal $T_{\text {aux }} \rightarrow 0$ and $T^{*} \rightarrow 2.8 \times 10^{-5}$ which corresponds

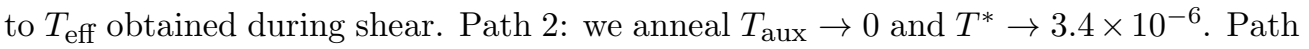
3: we anneal $T_{\text {aux }} \rightarrow 0$ but keep $T^{*}=3.4 \times 10^{-4}$ constant. When we set $T^{*}=T_{\text {eff }}$ (Path 1), the final elastic compressional energy value when $T_{\text {aux }} \rightarrow 0$ is very close to the mean value of the elastic energy obtained during shear $\langle E\rangle$. The value of the mean energy during shear is obtained for the same system as used in the annealing calculations. This demonstrates that $T_{\text {eff }}=X_{E}$ under the numerical accuracy of the simulations. For other values of $T^{*} \neq T_{\text {eff }}$ the final $E$ falls out of the distribution obtained during shear (Paths 2 and 3 ). We also follow different trajectories (not shown in the figure) to $T^{*} \rightarrow 2.8 \times 10^{-5}, T_{\mathrm{aux}} \rightarrow 0$ and find the same results indicating that our procedure is independent of the annealing path.

Annealing $T_{\text {aux }}$ to zero selects the jammed configurations $\left(E_{\text {jammed }}=0\right)$, while $T^{*}$ fixes the energy $E$.

In practice we perform equilibrium MD simulations with a modified potential energy:

$$
U=\frac{T_{\text {aux }}}{T^{*}} E+E_{\text {jammed }}
$$

and calculate the force on each particle from $\vec{F}=-\vec{\nabla} U$. Since we need to calculate the force from a potential energy, only conservative systems can be studied with this method. Thus we focus our calculations on the system of frictionless particles. The 
auxiliary temperature $T_{\text {aux }}$ is controlled by a thermostat which adjusts the velocities of the particles to a kinetic energy determined by $T_{\text {aux }}$. We start by equilibrating the system at high temperatures ( $T_{\text {aux }}$ and $T^{*} \sim \infty$ ) and anneal slowly the value $T_{\text {aux }}$ to zero and tune $T^{*}$ so as to reach the value of $E$ that corresponds to the average deformation energy obtained during shear.

The partition function is

$$
Z=\sum_{\nu} \exp \left[-E^{\nu} / T^{*}-E_{\text {jammed }}^{\nu} / T_{\text {aux }}\right],
$$

from where we obtain the compactivity as

$$
T^{*}=\frac{\partial E}{\partial S} \stackrel{T_{\text {aux }} \longrightarrow 0}{\longrightarrow} X_{E},
$$

Thus at the end of the annealing process $\left(T_{\text {aux }} \rightarrow 0\right), T^{*}(E)=X_{E}(E)$, since in this limit we are sampling the configurations with vanishing fraction of moving particles at a given $E$.

At the end of the protocol the compactivity at a given deformation energy can be obtained as illustrated in Fig. 9. The remarkably result is that the compactivity and the effective temperature obtained dynamically are found to coincide to within the computational error [23,

$$
X_{E} \approx T_{\text {eff. }} \text {. }
$$

This suggests the validity of the effective temperature as a dynamical estimate of the compactivity, and more importantly, justifies the use of the novel statistical measurements we have presented in characterising the macroscopic properties of the system.

\section{Conclusions}

We have experimentally tested the existence of the effective temperature for sheared granular system for a given range of particle sizes and densities. It remains to be seen how robust our results are under a more extended set of parameters. These include the use of particles of different shapes, changing the interstitial liquids, etc. It would also be important to test whether the effective temperature of the packing is the same under different types of driving, for instance under tapping or shaking, and for different observables. If the effective temperature is a proper state variable it should be independent of the observable. Experimentally it would require to measure the temperature from, for instance, the volume fluctuations. In fact an estimation of the compactivity as $X=\partial V / \partial S$ has been recently obtained in a system of compressed emulsions observed under the confocal microscope by Brujić et al. 41. It is still an open question what is the relation between the compactivity obtained from volume fluctuations and the effective temperature obtained from energy considerations. More experimentation is needed to fully understand whether the present definition of effective temperature has a physical thermodynamic meaning. Our results are a first step forward in this direction. 
Computer simulations shed some light on the thermodynamic meaning of the effective temperature. The fact that slow relaxation modes can be characterized by a temperature raises the question of the existence of a form of ergodicity for the structural motion, allowing a construction of a statistical mechanics ensemble for the slow motion of the grains. This argument leads us back to the ideas of the thermodynamics of jammed states. In parallel to these dynamical measurements obtained numerically and experimentally, the same information is drawn from the system by a flat statistical average over the jammed configurations done numerically. Once a sample of the static configurations have been visited by the system, the compactivity $X_{E}$ can be calculated from the statistics of the canonical ensemble of the jammed states. The logarithm of the available configurations at a given energy and volume reveals the entropy, from which the compactivity is calculated. Our explicit computation shows that the temperature arising from the Einstein relation can be understood in terms of the configurational compactivity $X_{E}$ arising from the statistical ensemble of jammed states. This provides evidence for the validity of the thermodynamic approach. However, much more experimentation is needed to fully test the thermodynamics of grains.

In summary, our experimental and numerical results suggest that the problem of slowly moving granular matter near jamming could be a nontrivial generalization of statistical mechanics to this kind of far-from-equilibrium dissipative system. 
[1] Liu, A. J. \& Nagel, S. R. (eds) (2001) Jamming and Rheology: Constrained Dynamics on Microscopic and Macroscopic Scales, (Taylor \& Francis, London).

[2] Mehta, A. \& Halsey, T. C. (eds), (2001) Proceedings of the Workshop "Challenges in Granular Physics" Adv. Complex Syst. 4, 287.

[3] A. Coniglio, A. Fierro, H. J. Herrmann, and M. Nicodemi (eds.) Unifying Concepts in Granular Media and Glasses (Elsevier, Amsterdam, 2004).

[4] Hinrichsen, H. \& Wolf, D. E. (eds) (2004) The Physics of Granular Media (Wiley-VCH Verlag).

[5] Grigera, T. S. \& Israeloff, N. E. (1999) Phys. Rev. Lett. 83, 5038.

[6] Bellon, L., Ciliberto, S. \& Laroche, C. (2001) Europhys. Lett. 53, 511.

[7] Hérisson, D. \& Ocio, M. (2002) Phys. Rev. Lett. 88, 257202.

[8] D’Anna, G., Mayor, P., Barrat, A., Loreto, V. \& Nori, F. (2003) Nature 424, 909.

[9] Ojha, R. P., Lemieux, P.-A., Dixon, P. K., Liu, A. J. \& Durian, D. J. (2004) Nature 427, 521.

[10] Edwards, S. F. The role of entropy in the specification of a powder, in Granular Matter: an Interdisciplinary Approach (Mehta, A., editor) (1994) 121-140 (Springer-Verlag, New York).

[11] H. A. Makse, J. Brujić and S. F. Edwards, in The Physics of Granular Media, H. Hinrichsen and D. E. Wolf, editors (Wiley-VCH Verlag, 2004).

[12] Nowak, E. R., Knight, J. B., Ben Naim, E., Jaeger, H. M. \& Nagel, S. R. (1998) Phys. Rev. E 57, 1971.

[13] Philippe, P. \& Bideau, D. (2002) Europhys. Lett. 60, 677.

[14] Chakravarty, A., Edwards, S. F., Grinev, D. V., Mann, M., Phillipson, T. E. \& Walton, A. J. Proceedings of the Workshop on Quasi-static Deformations of Particulate Materials, private communication.

[15] Edwards, S. F. (2004) J. Stat. Phys. 116, 29-42.

[16] Bideau, D., Philippe, P., Ribière, P. and Richard, P., in Unifying Concepts in Granular Media and Glasses, A. Coniglio, A. Fierro, H. J. Herrmann, \& M. Nicodemi (eds.) (Elsevier, Amsterdam, 2004), p63-76.

[17] Cugliandolo, L. F., Kurchan, J. \& Peliti, L. (1997) Phys. Rev. E 55, 3898.

[18] Kurchan, J. (2000) J. Phys. Condensed Matter 29, 6611.

[19] Langer, S. A. \& Liu, A. J. (2000) Europhysics Lett. 49, 68.

[20] Barrat, A., Kurchan, J., Loreto, V. \& Sellitto, M. (2000) Phys. Rev. Lett. 85, 5034.

[21] Ono, I. K., O'Hern, C. S., Durian, D. J., Langer, S. A., Liu, A. J. \& Nagel, S. R. (2002) Phys. Rev. Lett. 89, 095703.

[22] Fierro, A., Nicodemi, M. \& Coniglio, A. (2002) Europhys. Lett. 59, 642-647; (2002) Phys. Rev. E $66,061301$.

[23] Makse, H. A. \& Kurchan, J. (2002) Nature 415, 614.

[24] C. M. Song, P. Wang, and H. A. Makse, to appear in PNAS (2004).

[25] F. Potiguar and H. A. Makse, (submitted to Phys. Rev. E, 2004).

[26] Nedderman, R. (1992) Statics and Kinematics of Granular Materials (Cambridge Univ. Press, Cambridge).

[27] Drake, T. G. (1990) J. Geophys. Res. 95, 8681.

[28] Mueth D. M., Debregeas, G. F., Karczmar, G. S., Eng, P. J., Nagel, S. R. \& Jaeger, H. M. (2000) Nature 406, 385.

[29] Veje, C. T., Howell, D. W. \& Behringer, R. P. (1999) Phys. Rev. E 59, 739.

[30] Utter, B. \& Behringer, R. P. (2004) Phys. Rev. E 69, 031308.

[31] Weeks, E. R., Crocker, J. C., Levitt, A. C., Schofield, A. \& Weitz, D. A. (2000) Science 287, 627.

[32] Khosropour, R., Zirinsky, J., Pak, H. K., Behringer, R. P. (1997) Phys. Rev. E 56, 4467.

[33] Drahun, J. A. \& Bridgwater, J. (1983) Powder Technol. 36, 39.

[34] Jaeger, H. M., Nagel, S. R. \& Behringer, R. P. (1996) Rev. Mod. Phys.68, 1259.

[35] Tardos, G. I, McNamara, S. \& Talu, I. (2003) Powder Tech 131 23-39.

[36] Debenedetti, P. G. \& Stillinger, F. H. Nature 410, 259-267 (2001).

[37] Feitosa, K. \& Menon, N. (2002) Phys. Rev. Lett. 88, 198301. 
[38] P. A. Cundall, O. D. L. Strack, Géotechnique 29, 47 (1979).

[39] H. A. Makse, D. L. Johnson, L. M. Schwartz, Phys. Rev. Lett. 84, 4160 (2000).

[40] C. S. O'Hern, S. A. Langer, A. J. Liu, and S. R. Nagel, Phys Rev. Lett. 86, 111 (2001).

[41] S. F. Edwards, J. Brujić, and H. A. Makse, in Unifying Concepts in Granular Media and Glasses, A. Coniglio, A. Fierro, H. J. Herrmann, \& M. Nicodemi (eds.) (Elsevier, Amsterdam, 2004), p9-23.

We are deeply grateful to M. Shattuck for help in the design of the experiments and J. Kurchan and J. Brujić for discussions. We acknowledge financial support from the DOE, Division of Materials Sciences and Engineering, DE-FE02-03ER46089, and the National Science Foundation, DMR-0239504. 